\title{
Surface evolution of lanthanum strontium cobalt ferrite thin films at low temperatures
}

\author{
D. Newby Jra,*, J. Kuyyalil ${ }^{\mathrm{a}}$, J. Laverock ${ }^{\mathrm{a}}$, K. F. Ludwig ${ }^{\mathrm{a}}, \mathrm{Y} . \mathrm{Yu}^{\mathrm{c}}$,

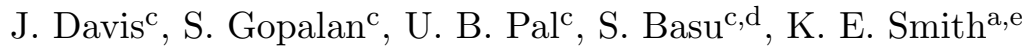 \\ ${ }^{a}$ Department of Physics, Boston University, 590 Commonwealth Avenue, Boston, MA \\ 02215, USA \\ ${ }^{b}$ Division of Materials Science and Engineering, Boston University,15 St. Mary's Street, \\ Brookline, MA 02446, USA \\ ${ }^{c}$ Division of Materials Science and Engineering, Boston University,15 St. Mary's Street, \\ Brookline, MA 02446, USA \\ ${ }^{d}$ Department of Mechanical Engineering, Boston University, 110 Cummington Street, \\ Boston, MA 02215, USA \\ ${ }^{e}$ School of Chemical Sciences and The MacDiarmid Institute for Advanced Materials and \\ Nanotechnology, The University of Auckland, Auckland 1142, New Zealand
}

\begin{abstract}
The ultra-high vacuum surface preparation of heteroepitaxial lanthanum strontium cobalt ferrite thin films has been studied using soft x-ray photoelectron spectroscopy. Specifically, the effect of annealing the films at low temperatures in low partial pressures of oxygen and argon has been investigated. We find that atmospheric surface carbon contamination of the films can be removed in select anneal temperature regimes in argon, but remains bound to the surface with oxygen annealing at any temperature. Irrespective of the gas used, an insulating phase transition occurs near $300{ }^{\circ} \mathrm{C}$ due to strontium segregation at the surface. The surface develops more insulating character if annealed with oxygen. Different species are proposed to be responsible for the discrepancy in insulating character.
\end{abstract}

Keywords: X-ray photoemission spectroscopy, Solid Oxide Fuel Cell Cathodes, Thin Films, Perovskite Cathode Materials, Lanthanum Strontium Cobalt Ferrite

\footnotetext{
*Corresponding author

Email address: dnewby@bu.edu (D. Newby Jr)
} 


\section{Introduction}

In the continued efforts to improve energy resource utilization for the twenty-first century, few technologies hold as much promise as solid oxide fuel cells (SOFCs) [1, 2]. A diagram of fuel cell operation is shown in Fig.

51 . On the left, fuel in the form of hydrocarbons is fed into the cell, where it thermally cracks into a number of products. Among those products is hydrogen, which reacts with oxygen ions at the anode. The oxygen ions are provided by the cathode, which reduces oxygen from air. The electrolyte is an ion-conducting material that provides a path for the oxygen radicals.

10 Water is produced at the anode. Usable electric current is produced by the potential difference between the electrodes.

Perovskite-based fuel cell devices can reliably generate electrical power from a wide variety [3, 4] of fossil fuels, using existing infrastructure and with high energy density in a small volume. Critical to the operation of a

15 SOFC is the cathode material, which produces oxygen ions and transports them into the electrode. Indeed, cathode surface and interface modification is one of the primary contributors $[5,6]$ to the operational degradation of an operating fuel cell. Understanding the processes at the cathode is critical to potential improvements and elucidating the factors limiting the life of these devices.

Many high-temperature fuel cells use lanthanum-doped strontium manganite (LSM) cathodes [7], the reaction kinetics of which are limited to the triple-phase boundary [8]. Overcoming the limitations imposed by the triplephase boundary would allow for higher energy densities and efficiencies.

25 Mixed ion-electron conductors can transport oxygen ions using more of their surface area, without relying on the electrode interfaces. One of the most promising mixed ion-electron conductors under popular investigation for use as a cathode is lanthanum strontium cobalt ferrite $\left(\mathrm{La}_{0.6} \mathrm{Sr}_{0.4} \mathrm{Co}_{0.2} \mathrm{Fe}_{0.8} \mathrm{O}_{3-\delta}\right.$, abbreviated LSCF-6428). LSCF offers high electron and ion conductivity, as well as good compatibility with other standard SOFC construction materials [9]. This combination of features may be useful in engineering lowtemperature fuel cells with the performance necessary for more widespread application.

Since the surface structure and composition of real cathodes determine many of the performance attributes of fuel cells, investigations into cathode surface evolution give valuable insight towards improvement. Soft x-ray spectroscopy can provide element-specific chemical environment information for shallow sampling depths- precisely the region at play during operation. Previous x-ray studies have observed strontium segregation at the surface 
of LSCF films during operational-temperature annealing in air [10]. Similar effects have been observed in other strontium-based perovskite cathode materials [11]. The segregation of strontium and its oxides/carbonates at the surface of perovskite films has been shown $[5,12]$ to decrease the sites available for oxygen catalysis as well as retarding electron transport to the also been shown that rapidly quenching the temperature of cathode films can preserve surface configuration changes, which allows for post-anneal or post-operation measurement [10].

Critically, exposure to atmospheric contaminants may interfere with surface-sensitive techniques, and surface treatment (e.g. degassing and cleaning) is necessary [13] for conventional soft x-ray measurements. In the case of perovskite thin films the effects of contamination are likely exacerbated due to the enhanced catalytic activity observed [14]. To that end, a study of the low-temperature surface evolution is of interest for future surface studies toemission spectroscopy to determine the evolving chemical states on the surface of an LSCF film. Using very low $\left(\sim 10^{-8}\right.$ torr $)$ partial pressures, we demonstrate that existing surface carbon can be removed from the surface via annealing in argon. This technique produces a clean, defect-free film. Using similar pressures of oxygen during anneal cycles has drastically different results, leaving surface carbon bound to the surface and bringing on a relatively strong insulating surface phase transition. These results serve as a template for clean surface preparation of LSCF thin films, useful for further investigation into the surface interactions of these interesting materials.

\section{Materials and Methods}

Pulsed laser deposition was used to grow epitaxial films of LSCF-6428 on $\mathrm{NdGaO}_{3}$ substrates, with the orientations (001) and (110), respectively. The films were deposited using an LSCF target, at an oxygen partial pressure of 10 mTorr with the substrate temperature at $550{ }^{\circ} \mathrm{C}$. Films of approximately $250 \mathrm{~nm}$ thickness were produced. Characterization via atomic force microscopy, x-ray diffraction, and transmission electron microscopy confirmed the film quality and orientation. The mean surface roughness was measured at $0.68 \mathrm{~nm}$. All film deposition and characterization was conducted at the Environmental Molecular Sciences Laboratory, Pacific Northwest National Laboratory.

Soft x-ray spectroscopy measurements were performed at the soft xray undulator beamline X1B at the National Synchrotron Light Source, 
Brookhaven National Laboratory. Ultra-high vacuum (UHV, $<10^{-10}$ torr) was maintained in the experimental chamber during data acquisition. X-ray photoemission spectroscopy (XPS) was performed using a hemispherical analyzer and monochromated incident x-rays at 250 and $900 \mathrm{eV}$. The total resolution of these spectra is approximately $0.4 \mathrm{eV}$ full-width at half-maximum (FWHM) at the Sr $3 d$ edge, and $0.7 \mathrm{eV}$ FWHM at the oxygen and carbon $K$-edges. Binding energies were calibrated with reference to the $4 f$ core levels of clean gold foil in electrical contact with the sample. In order to probe the surface chemistry of these materials, the soft x-rays employed are primarily surface-sensitive, and by varying the relative angle between the film surface and the photoelectron spectrometer, it is possible to tune the depth sensitivity for photoelectron spectroscopy. This has been used here to differentiate between surface and bulk chemical species.

After sonication in acetone, samples were mounted on tantalum foil, with the film grounded by tantalum strips. Samples were radiatively heated through the substrate using a bare tungsten filament. The power supplied to the filament was monitored using a constant voltage/current power supply. thermocouple in contact with the surface.

Precautions were taken to ensure that chromium contamination, originating from the K-type thermocouple alloy, did not affect the films. The calibration was completed after XPS data were taken for all anneals, in or100 der to avoid the thermocouple (TC) affecting the surface. While chromium poisoning is a well-known vulnerability of $\operatorname{LSCF}[8,15,16]$, it is worth noting that no chromium features were observed in XPS survey scans taken after the TC-contact calibrations. This suggests that chromium poisoning is not a concern at the temperatures used here, which is in reasonable agreement 105 with thermodynamic calculations performed [15] for other perovskites. Contact thermocouple temperature measurement may be utilized in this regime without negative consequence.

High-purity oxygen and argon were selectively dosed through a constant pressure valve $\left(10^{-8}\right.$ torr $)$ with continuous pumping in the chamber, creating 110 a steady supply of fresh gas across the sample surface. Oxygen was chosen for its role in the operational environment of SOFCs, and argon in order to provide contrast as an inert gas at the same partial pressures. For each anneal cycle, dosing and heating were combined for a period of time resulting in 50 Langmuir of total exposure. The samples were then quenched via abrupt power cutoff to the filament, and allowed to cool to room temperature in their respective annealing gases. The preparation chamber was then evacuated, and the film moved into a measurement chamber maintained at 
$10^{-10}$ torr.

\section{Results and Discussion}

\subsection{Carbon $1 s$}

Once a film has been exposed to air, it is subject to a number of contaminating adsorbents. For the present system the most notable contaminant is carbon in the form of atmospheric carbon dioxide; carbonates will tend to form [17] on surface strontium and its oxides. Since the measurement chamber is maintained under UHV, surface carbon carried in by the film can become a prime source of carbonate formation in addition to unavoidable carbon species already present in the chamber. An excess of surface carbon and its derivatives could potentially modify the surface chemistry in ways unrepresentative of the operating conditions we seek to mimic; it is therefore worthwhile to monitor the evolution of signal from carbon as the experiment proceeds.

The extent of carbon contamination on the surface can be directly studied through measurement of the carbon $1 s$ core level. The relative height of the C $1 s$ XPS peak near $285 \mathrm{eV}$ compared to the strontium $3 p$ peaks near $268 \mathrm{eV}$ and $278 \mathrm{eV}$ gives a good indication of how much carbon is adsorbed on the surface, since no carbon should be included in the bulk LSCF film. Figure 1 shows the core-level scans in the region of $\mathrm{C} 1 s$, for successive anneals in argon at $10^{-8}$ torr.

\subsubsection{Argon Atmosphere Annealing}

Figure 2 shows the photoemission spectra taken after each annealing temperature for anneals in an argon partial pressure. The spectra show the C $1 s$ peak at binding energy of $284.6 \mathrm{eV}$ (with dotted line as a guide to the eye), as well as the strontium $3 p$ doublet at $268.4 \mathrm{eV}$ and $278.7 \mathrm{eV}$. It is clear that as the annealing temperature increases, the intensity of the $\mathrm{C}$

$1 s$ peak reduces in intensity. This suggests that the contaminant carbon desorbs from the surface as the temperature is increased, most significantly above $150{ }^{\circ} \mathrm{C}$. Above $200{ }^{\circ} \mathrm{C}$, contaminant carbon on the surface is below our detection limit.

Above $245{ }^{\circ} \mathrm{C}$, an additional species develops on the sample surface, present as a doublet-like structure at 294-296 eV. After ruling out other contaminant elements, we identify this structure with chemically shifted carbon. This suggests the formation of new species as a result of carbon re-adsorption on the surface. The lack of strontium carbonate features (see Section 3.3) suggests that carbon is bonding to other elements present in 
the sample; in the current data set, the precise bonding environment of the re-adsorbed carbon species cannot be fully determined.

In summary, annealing LSCF in low partial pressure argon provides a route for a carbon-free surface, up to a certain temperature. The formation of carbonate species from ambient carbon cannot be avoided over $245^{\circ} \mathrm{C}$ at these pressures in argon.

\subsubsection{Oxygen Atmosphere Annealing}

From Fig. 3 it can be seen that with increasing annealing temperature in oxygen there is a prominent reduction in the intensity of the $\mathrm{C} 1 s$ peak, but not to the degree shown during argon anneals. In a pure oxygen atmosphere, a small fraction of surface carbon continues to persist throughout the anneal cycles. This is in addition to the re-adsorbed carbonate doublet, similar to that observed in argon anneals, which forms at higher temperatures $(>260$ ${ }^{\circ} \mathrm{C}$ ) for oxygen anneals.

Even at very low partial pressures, an ambient oxygen atmosphere inhibits the removal of contaminant carbon from pristine LSCF film surfaces. For future surface studies that require clean LSCF, this result is of great practical use.

\subsection{Oxygen $1 \mathrm{~s}$}

\subsubsection{Argon Atmosphere Annealing}

Since the operative function of a SOFC cathode is to reduce oxygen and move it into an electrolyte, observing the evolution of surface oxygen states is of primary importance in this investigation. Of particular interest is the formation of surface oxygen vacancies, which play an important role in operational cathode catalysis dynamics [8]. Site vacancies can be inferred and quantified from photoemission core level peaks $[18,19]$. In this section the O $1 s$ core levels are observed with increasing anneal temperature, following the procedure above. The binding energies for pristine sample fitted peaks were $528.8 \mathrm{eV}$ and $531.5 \mathrm{eV}$ for the bulk and surface, respectively. The bulk peak maintained its position throughout anneals in oxygen and argon, whereas the surface peak showed a gradual shift in spectral weight of $0.5 \mathrm{eV}$ with temperature increase.

Figure 4 shows the results obtained for the argon-annealed film. Figure 4(a) displays the $\mathrm{O} 1 s$ spectrum for each successive anneal, where the temperature is increased from $98{ }^{\circ} \mathrm{C}$ to $290{ }^{\circ} \mathrm{C}$. It is apparent that the $\mathrm{O} 1 \mathrm{~s}$ spectra show prominent changes with increase in anneal temperature. We performed multi-peak fitting on the spectra, representative results of which 
are shown in the right side panel. Figures 4(b), (c) and (d) illustrate fits at room temperature, and at annealing temperatures of $220{ }^{\circ} \mathrm{C}$ and $278{ }^{\circ} \mathrm{C}$.

The multi-peak fits here were conducted using Voigt lineshapes with a

Shirley background. The O $1 s$ spectra from pristine sample can be fitted with two components at binding energies of $528.5 \mathrm{eV}$ and $531.5 \mathrm{eV}$. Varying the orientation of the film surface relative to the XPS spectrometer allows for selective depth profiling, from which it can be shown that the component at low binding energy originates from the bulk and the higher binding component is from surface layers. The two components will be denoted as bulk and surface components for further discussion. At higher annealing temperatures a further component appears and the fitting of the spectra requires three components as shown in Figs. 4(b) and (c). The angle-dependent measurements show that this species is uniformly distributed in the surface and the bulk. Since it only arises during heating, and increases nearly linearly with temperature, it is likely the result of oxygen defects (vacancies) resulting from heating in an inert atmosphere.

To extract the evolution of each component of the O $1 s$ core level spectra as the annealing temperature is increased, it is useful to examine the fractional area (individual area/total area) of each component during the experiment; these results are plotted in Fig. 5. Up to $\sim 150{ }^{\circ} \mathrm{C}$ each curve in Fig. 5 shows a plateau, indicating that the area of each component remains unchanged even with increases in annealing temperature, and suggesting that the relative concentrations of oxygen species do the same. Above this temperature, the component corresponding to vacancies increases almost linearly with temperature, showing the increase of defects in the material with increasing temperature. Above $150{ }^{\circ} \mathrm{C}$ there is a sharp rise in the area of the bulk component, which is accompanied by a sharp fall in the area of surface component. Between $150{ }^{\circ} \mathrm{C}$ and $175{ }^{\circ} \mathrm{C}$ there is an inversion of the surface:bulk character ratio. This can be correlated with observations at the strontium $3 d$ edge (see Section 3.3), suggesting that the oxygen vacancies created through heating cause changes in the Sr-O bonding environment. Above $175{ }^{\circ} \mathrm{C}$ the area of the surface component increases approximately linearly and that of bulk component linearly decreases. Judging by these trends, the vacancies are forming at the expense of oxygen in the bulk. The surface oxygen, after a certain temperature has been reached, returns to its initial state and concentration.

\subsubsection{Oxygen Atmosphere Annealing}

The $\mathrm{O} 1 s$ core levels are similarly followed during oxygen anneals. Figure 6(a) shows the O $1 s$ core-level spectra for successive anneals in oxygen. In 
contrast to annealing in argon, the changes to the $\mathrm{O} 1 \mathrm{~s}$ spectra are sudden and drastic for anneals in oxygen, as evidenced by the accompanying multipeak fits. The representative fitting curves are shown in Figs. 6(b), (c) and (d) which show spectra for pristine films, and films annealed at temperatures 235 of $220^{\circ} \mathrm{C}$ and $278{ }^{\circ} \mathrm{C}$ respectively. As in the case of annealing in argon, the spectra can be fitted with 3 components: one bulk, one surface, and a third that only develops upon heating in oxygen. It is important to note that this third species, induced by heating in a partial oxygen atmosphere, is not the same as the vacancy environment observed with argon anneals; it 240 arises at an altogether different binding energy. The fractional area of each component is shown against the annealing temperature in Fig. 7. In the case of $\mathrm{O} 1 \mathrm{~s}$, the fitted surface component shifts its spectral weight by .7 $\mathrm{eV}$ at higher temperatures; the bulk peak remains the same. The vacancy environment peak shifts by approximately $.2 \mathrm{eV}$ at higher temperatures, which may indicate the onset of a surface phase change.

Comparing Figs. 5 and 7 it can be seen that the there are prominent differences between the areas of different $\mathrm{O} 1 s$ components depending on the annealing gas used. In oxygen (Fig.7), as the temperature is increased, the area of the third species increases linearly and roughly saturates above $150{ }^{\circ} \mathrm{C}$. This corresponds to the behavior one would expect of oxygen defect environments at low partial pressures; defects are formed in the surface and bulk, but eventually reach equilibrium with the replenishment provided by the ambient oxygen.

Following the above considerations, the formation of vacancies is inhibited in the presence of oxygen at high temperatures, which is quite unlike the behavior during annealing in argon. Above $100{ }^{\circ} \mathrm{C}$ the bulk component decreases with temperature, which is accompanied by a corresponding increase in the surface component. The increasing surface-like character of the film may be a result of carbon surface reaction (discussed above), the kinetics of which are clearly more favorable for a partial oxygen atmosphere, and carbonate formation, which still takes place at higher temperatures. The difference in third environment between inert gas (argon) and oxygen anneals can be accounted for by the surface incorporation of carbon; the environment indicative of an oxygen vacancy is altered by the persistent 265 presence of carbon.

The stabilization of oxygen vacancies by low oxygen partial pressure was recently predicted theoretically by Luo et al. [20]. Our results in both cases support that work, albeit at much lower pressures than discussed in Ref. $[20]$. 


\subsection{Strontium 3d}

Strontium precipitation in its oxide and carbonate forms is widely accepted $[5,21,22]$ to be one of the influences behind operational degradation of LSCF cathodes. Studies have shown conclusively that strontium precipitates are energetically favorable in these films when they are held in air (or other atmosphere-pressure gases) at high temperatures $\left(>600{ }^{\circ} \mathrm{C}\right)$ for long periods of time.

Figure 8 shows a typical Sr $3 d$ core level. The $3 d$ level is a doublet, with two peaks spaced $1.8 \mathrm{eV}$ apart due to the spin-orbit split $3 d_{3 / 2}$ and $3 d_{5 / 2}$ states [23]. Good agreement with the data is achieved by fitting this structure to a pair of Gaussian doublets and a linear background. In this fit, the energy separation and relative intensities of the peaks within the doublet are fixed, and only the relative intensity between the two doublets and their energy separation is allowed to vary. Varying the relative orientation of the films surface with respect to the spectrometer allows for relative depth profiling (as above), and in doing so the lower binding energy doublet can be associated with the bulk, and the higher binding energy doublet is determined to originate at the surface.

Fitting the peaks to identify the surface and bulk phases allows for the observation of changes in the strontium concentration after anneal cycling. Figure 9 shows the fractional area of the bulk and surface components, plotted against the temperature at which the sample was annealed. Error bars corresponding to the statistical uncertainty in the fit are shown at each point. At grazing incidence, these measurements are more surface sensitive; this is in good agreement with the fitted intensities for the bulk and surface components. Clear changes are visible throughout the heating process, as well as stark differences in the surface evolution between argon and oxygen anneals.

The integrated doublet areas are plotted as fractions of the total area of the fit in Fig. 9. Notably, the first points for the normal incidence behavior do not match, but they do for grazing incidence. This suggests that the surfaces for the respective samples are the same, but there is some difference in the bulk.

The above results can be explained by the specific geometry of the data acquisition chamber. The XPS collection aperture is $45^{\circ}$ offset from the incoming x-rays. Rotating the sample increases the surface sensitivity due to the geometry of primary photoelectrons leaving the samples. Simultaneously the sampling area visible to the XPS aperture increases by a factor of $1 / \cos (\theta)$. At grazing incidence for the data presented here, the sampling area is larger by over $30 \%$. Local inhomogeneities in the film surface 
due to atmospheric contamination will therefore be more apparent at normal incidence, since the larger sampling area for grazing incidence will tend to average them out. This accounts for the difference in initial conditions present at normal incidence.

Certainly, prior to any heating at low pressure, the films can be expected to have slightly different surface contamination levels, in spite of identical cleaning and handling before insertion into UHV. After the film is gently annealed at $100{ }^{\circ} \mathrm{C}$, but before surface carbon is strongly affected, the normal incidence bulk and surface areas are nearly the same. This supports the hypothesis that local differences in adsorbed atmospheric gases cause the initial difference.

Turning to the oxygen anneals (displayed by the solid lines in Fig. 9), it is worth noting that there are several distinct regions of change. For heating $\leq 100{ }^{\circ} \mathrm{C}$, any visible change is likely due to the degassing of the film (visible in the carbon spectra presented above). Past the temperature at which carbon concentration begins to decrease at the surface, there is a clear trend of increase in the surface strontium concentration.

The argon anneal fractional areas (dotted lines) behave quite differently. Between the first and second heatings the bulk and surface phases behave in the opposite way to their oxygen-annealed counterparts. This is the interval during which the majority of carbon contamination is seen to leave the surface. Past the second anneal, the argon-annealed surface goes through an abrupt change in direction and begins to develop towards the trend observed during the oxygen anneal. Notably, above $200{ }^{\circ} \mathrm{C}$, argon annealing can return the strontium surface concentration close to its original configuration.

Eventually, near $300{ }^{\circ} \mathrm{C}$, both types of annealed surfaces end up with nearly the same surface:bulk concentrations. Clearly not all of the carbon has been evacuated from the surface on the oxygen-annealed sample, as shown in the carbon core levels. Additionally, the oxygen-annealed sample goes through a phase transition to relatively strong insulating behavior (see section 3.4), which is not visible in the argon-annealed sample. Both samples, notably, see an increase in surface-phase strontium concentration.

No contributions from $\mathrm{SrCO}_{3}$ are seen in the strontium core levels. If carbonates form on the surface at these temperatures, they should [24] be visible as an additional doublet in the strontium spectra. The absence of such a feature suggests that strontium does not form its carbonate phase at these temperatures and pressures. 


\subsection{Insulating Surface Phase}

For both annealing gases employed in this study, we find that the films undergo a phase transition that promotes an insulating character at the surface near $300{ }^{\circ} \mathrm{C}$. This insulating transition can be observed in timedependent XPS scans, whereby the charging of the insulating surface develops with time and leads to large shifts in the apparent binding energies of core-level features. It is observed that argon-annealed films enter a less insulating state, even when annealed to higher temperatures.

Core level electrons from a conducting sample should have the same measured kinetic energy over time, assuming that the incident photon energy is constant and the originating core environment does not change. If the surface of a sample is gradually accumulating charge, the kinetic energy of a core level peak will shift in proportion to the total charge on the surface. Since electrons leave the surface, an overall positive charge will evolve in the sample, which in turn results in a net decrease in the kinetic energy of electrons emanating from the surface. Ultimately, this leads to an increase in the observed binding energy of core levels for samples that are not good conductors.

Figure 10 shows select time-dependent measurements of the Sr $3 d$ core level for both samples. Fig. 10(a) shows the time-integrated intensity for a sample that does not exhibit charging. Fig. 10(b) shows a scan from a sample annealed in oxygen to $246{ }^{\circ} \mathrm{C}$. Time proceeds from top to bottom, with each horizontal scan taking approximately 30 seconds. In the first color map, the horizontal (binding energy) position doesn't change appreciably with time. In Fig. 10(c), the binding energy of the peak clearly changes over time. This figure shows the same sample as in Fig. 10(b), but annealed to $260^{\circ} \mathrm{C}$ in oxygen. The discrepancy in the location on the horizontal scales is due to the fact that the second scan was acquired after the sample had already been exposed to the x-ray flux for some time, leading to a substantial accumulation in charge before the second measurement took place.

In contrast, Figs. 10(d) and (e) show the Sr $3 d$ peaks of a sample annealed in argon at even higher temperatures. Above the point where the oxygen-annealed sample develops a surface insulating state that severely hampers the XPS measurements, the argon-annealed sample has no such transition. At higher temperatures the argon anneal appears to produce some insulating character, but not to the same severity that annealing in oxygen does. Below $300{ }^{\circ} \mathrm{C}$, argon annealing mostly preserves the conducting character of the surface. Below $260{ }^{\circ} \mathrm{C}$, the conducting character is entirely preserved. 


\section{Conclusions}

It has been shown that low temperature, low partial pressure treatment of LSCF-6428 films can be used to remove contaminants and produce pristine films for UHV experimentation. Carbon contamination from atmospheric exposure can be selectively eliminated by annealing at $200{ }^{\circ} \mathrm{C}$ in an argon atmosphere or boundd to the surface using an anneal in oxygen. In either environment, carbonate formation proceeds above $245{ }^{\circ} \mathrm{C}$. Carbonates of strontium are not observed, suggesting that temperatures in this regime are too low to provoke their large-scale formation. The formation of surface environments attributable to oxygen vacancies is unavoidable with heating. These vacancies are observed to plateau in number when the anneal takes place in low oxygen partial pressure, but increase linearly with temperature when argon is used. Annealing in argon at temperatures of $200-245{ }^{\circ} \mathrm{C}$ produces a clean, carbon-free surface with oxygen vacancies.

\section{Acknowledgments}

Supported in part by the NSF under award CHE-1213381. Experiments performed at the National Synchrotron Light Source, Brookhaven National Laboratory, which is supported by the U.S. Department of Energy under Contract No. DE-AC02-98CH10886.

\section{References}

[1] E. T. S. Inc, Fuel Cell Handbook, 7th Edition, Business/Technology Books, 2004. doi:10.2172/834188.

[2] S. Singhal, Advances in solid oxide fuel cell technology, Solid State Ionics 135 (1-4) (2000) 305-313. doi:10.1016/S0167-2738(00)00452-5.

410 [3] G. Cinti, U. Desideri, D. Penchini, G. Discepoli, Experimental Analysis of SOFC Fuelled by Ammonia, Fuel Cells 14 (2) (2014) 221-230. doi:10.1002/fuce.201300276.

[4] A. C. Rady, S. Giddey, A. Kulkarni, S. P. Badwal, S. Bhattacharya, B. P. Ladewig, Direct carbon fuel cell operation on brown coal, Applied 415 Energy 120 (2014) 56-64. doi:10.1016/j.apenergy.2014.01.046.

[5] D. Oh, D. Gostovic, E. D. Wachsman, Mechanism of La0.6Sr0.4Co0.2Fe0.8O3 cathode degradation, Journal of Materials Research 27 (15) (2012) 1992-1999. doi:10.1557/jmr.2012.222. 
[6] A. Weber, E. Ivers-Tiffée, Materials and concepts for solid oxide fuel cells in stationary and mobile applications, Journal of Power Sources 127 (1-2) (2004) 273-283. doi:10.1016/j.jpowsour.2003.09.024.

[7] S. Singhal, K. Kendall (Eds.), High-temperature solid oxide fuel cells: fundamentals, design and applications, Elsevier, 2003.

[8] C. Sun, R. Hui, J. Roller, Cathode materials for solid oxide fuel cells: a review, Journal of Solid State Electrochemistry 14 (7) (2009) 11251144. doi:10.1007/s10008-009-0932-0.

[9] N. A. Baharuddin, H. A. Rahman, A. Muchtar, A. B. Sulong, H. Abdullah, Development of lanthanum strontium cobalt ferrite composite cathodes for intermediate- to low-temperature solid oxide fuel cells, Journal of Zhejiang University SCIENCE A 14 (1) (2013) 11-24. doi:10.1631/jzus.A1200134.

[10] J. Davis, L. Miara, L. Saraf, T. Kaspar, S. Gopalan, U. B. Pal, J. C. Woicik, S. N. Basu, K. Ludwig, Hard X-ray Fluorescence Measurements of Heteroepitaxial Solid Oxide Fuel Cell Cathode Materials, ECS Transactions 41 (11) (2012) 19-24.

[11] A. Monsen, F. Song, Z. Li, J. Boschker, T. Tybell, E. Wahlström, J. Wells, Surface stoichiometry of $\mathrm{La}_{0.7} \mathrm{Sr}_{0.3} \mathrm{MnO}_{3}$ during in vacuo preparation; A synchrotron photoemission study, Surface Science 606 (17-18) (2012) 1360-1366. doi:10.1016/j.susc.2012.04.018.

[12] Y. Chen, W. Jung, Z. Cai, J. J. Kim, H. L. Tuller, B. Yildiz, Impact of Sr segregation on the electronic structure and oxygen reduction activity of $\mathrm{SrTi}_{1 x} \mathrm{Fe}_{x} \mathrm{O}_{3}$ surfaces, Energy \& Environmental Science 5 (7) (2012) 7979. doi:10.1039/c2ee21463f.

[13] L. F. J. Piper, A. R. H. Preston, S.-W. Cho, A. DeMasi, B. Chen, J. Laverock, K. E. Smith, L. J. Miara, J. N. Davis, S. N. Basu, U. Pal, S. Gopalan, L. Saraf, T. Kaspar, A. Y. Matsuura, P.-A. Glans, J.H. Guo, Soft X-Ray Spectroscopic Study of Dense Strontium-Doped Lanthanum Manganite Cathodes for Solid Oxide Fuel Cell Applications, Journal of The Electrochemical Society 158 (2) (2011) B99. doi:10.1149/1.3519075.

[14] G. laO', S.-J. Ahn, E. Crumlin, Y. Orikasa, M. Biegalski, H. Christen, Y. Shao-Horn, Catalytic Activity Enhancement for 
Oxygen Reduction on Epitaxial Perovskite Thin Films for SolidOxide Fuel Cells, Angewandte Chemie 122 (31) (2010) 5472-5475. doi:10.1002/ange.201001922.

[15] H. Yokokawa, T. Horita, N. Sakai, K. Yamaji, M. Brito, Y. Xiong, H. Kishimoto, Thermodynamic considerations on $\mathrm{Cr}$ poisoning in SOFC cathodes, Solid State Ionics 177 (35-36) (2006) 3193-3198. doi:10.1016/j.ssi.2006.07.055.

[16] S. Taniguchi, M. Kadowaki, H. Kawamura, T. Yasuo, Y. Akiyama, Y. Miyake, T. Saitoh, Degradation phenomena in the cathode of a solid oxide fuel cell with an alloy separator, Journal of Power Sources 55 (1) (1995) 73-79. doi:10.1016/0378-7753(94)02172-Y.

[17] V. Young, T. Otagawa, XPS studies on strontium compounds, Applications of Surface Science $20 \quad$ (3) (1985) $228 \quad-248$. doi:http://dx.doi.org/10.1016/0378-5963(85)90083-2.

[18] K. S. a. Butcher, a. J. Fernandes, P. P.-T. Chen, M. WintrebertFouquet, H. Timmers, S. K. Shrestha, H. Hirshy, R. M. Perks, B. F. Usher, The nature of nitrogen related point defects in common forms of InN, Journal of Applied Physics 101 (12) (2007) 123702. doi:10.1063/1.2736654.

[19] A. K. Rumaiz, B. Ali, A. Ceylan, M. Boggs, T. Beebe, S. Ismat Shah, Experimental studies on vacancy induced ferromagnetism in undoped $\mathrm{TiO}_{2}$, Solid State Communications 144 (7-8) (2007) 334-338. doi:10.1016/j.ssc.2007.08.034.

[20] H. Luo, Y. Shin, Y. Yu, D. Cetin, K. Ludwig, U. Pal, S. N. Basu, S. Gopalan, X. Lin, Predicting oxygen vacancy non-stoichiometric concentration in perovskites from first principles, Applied Surface Science 323 (0) (2014) 65 - 70. doi:10.1016/j.apsusc.2014.06.098.

480 [21] F. Wang, K. Yamaji, D.-H. Cho, T. Shimonosono, H. Kishimoto, M. E. Brito, T. Horita, H. Yokokawa, Sulfur Poisoning on $\mathrm{La}_{0.6} \mathrm{Sr}_{0.4} \mathrm{Co}_{0.2} \mathrm{Fe}_{0.8} \mathrm{O}_{3}$ Cathode for SOFCs, Journal of The Electrochemical Society 158 (11) (2011) B1391. doi:10.1149/2.059111jes.

[22] E. Bucher, W. Sitte, Long-term stability of the oxygen exchange properties of ( $\mathrm{La}, \mathrm{Sr}) 1 \mathrm{z}(\mathrm{Co}, \mathrm{Fe}) \mathrm{O} 3 \delta$ in dry and wet atmospheres, Solid State Ionics 192 (1) (2011) 480-482. doi:10.1016/j.ssi.2010.01.006. 
[23] A. Thompson, et al., X-ray data booklet, Lawrence Berkeley National Laboratory, 2009.

[24] P. A. W. van der Heide, Systematic x-ray photoelectron spectroscopic study of $\mathrm{La}_{1-x} \mathrm{Sr}_{x}$-based perovskite-type oxides, Surface and Interface Analysis 33 (5) (2002) 414-425. doi:10.1002/sia.1227. 


\section{List of Figures}

1 (Color available online) The operating principle of a solid oxide fuel cell. . . . . . . . . . . . . . . . . . . . 18

(Color available online) XPS spectra showing C $1 s$ and $\mathrm{Sr}$ $3 p$ core levels after successive anneals in $10^{-8}$ torr partial pressure of argon. Surface carbon appears $\sim 285 \mathrm{eV}$; a dotted line is provided as a guide to the eye. . . . . . . . . . . 19

3 (Color available online) XPS spectra showing C $1 s$ and $\mathrm{Sr}$ $3 p$ core levels after successive anneals in $10^{-8}$ torr partial pressure of oxygen. Spectra are normalized to the Sr $3 p$ edge for comparison with Figure $2 \ldots \ldots$. . . . . . . . 20

4 (Color available online) Oxygen $1 s$ spectra (a) for argonannealed films. Typical multi-peak fits are shown in (b-d).Fits were performed using Voigt peaks with a Shirley background. 21

5 (Color available online) O $1 s$ core level multi-peak Voigt fit fractional areas, argon-annealed film. Solid lines are provided as guides to the eye. . . . . . . . . . . . . . 22

6 (Color available online) Oxygen $1 s$ spectra (a) for oxygenannealed films. Typical multi-peak fits are shown in (b-d).Fits were performed using Voigt peaks with a Shirley background. 23

$7 \quad$ (Color available online) O1 $s$ core level multi-peak Voigt fit fractional areas, oxygen-annealed film. Solid lines are provided as guides to the eye. . . . . . . . . . . . . . . 24

8 (Color available online) Typical strontium $3 d$ core level for LSCF-6428. Best fit for two Gaussian doublets with linear background plotted against data. Inset shows the determination of surface vs. bulk states by angular surface sensitivity increase; as the surface angle with respect to the spectrometer increases, the measurement is more surface sensitive. . . . . . 25

9 (Color available online) Fractional areas of the surface/bulk strontium phases over successive heating cycles, at (a) normal and (b) grazing incidence. Grazing incidence is more surfacesensitive. . . . . . . . . . . . . . 26 
10 (Color available online) Time-dependent scans of Sr $3 d$ core levels. Time per scan is approximately 45 seconds. From top to bottom: (a) A plot showing integrated intensity over time for a well-conducting sample. (b) A time-dependent scan of a film annealed in $\mathrm{O}_{2}$ at $246{ }^{\circ} \mathrm{C}$, in which time proceeds down the y-axis.(c) A time-dependent scan clearly showing charge buildup over time after $\mathrm{O} 2$ annealing at $260{ }^{\circ} \mathrm{C}$. (d),(e) Two more scans, of argon-annealed films annealed to over $278{ }^{\circ} \mathrm{C}$, showing little charge buildup. . . . . . . . . . . . . 27 


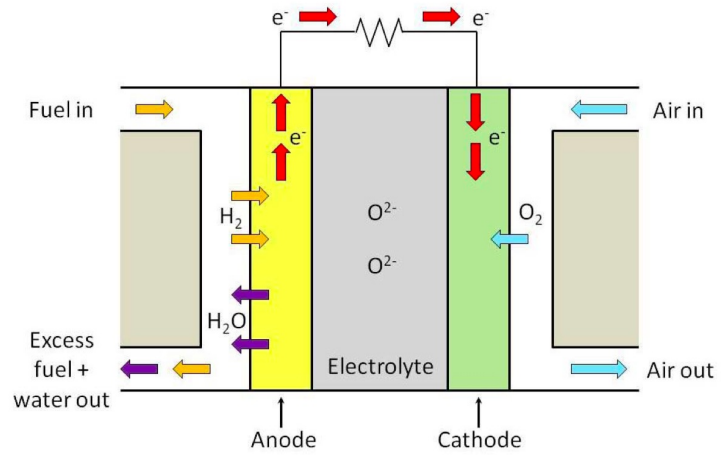

Figure 1: (Color available online) The operating principle of a solid oxide fuel cell. 


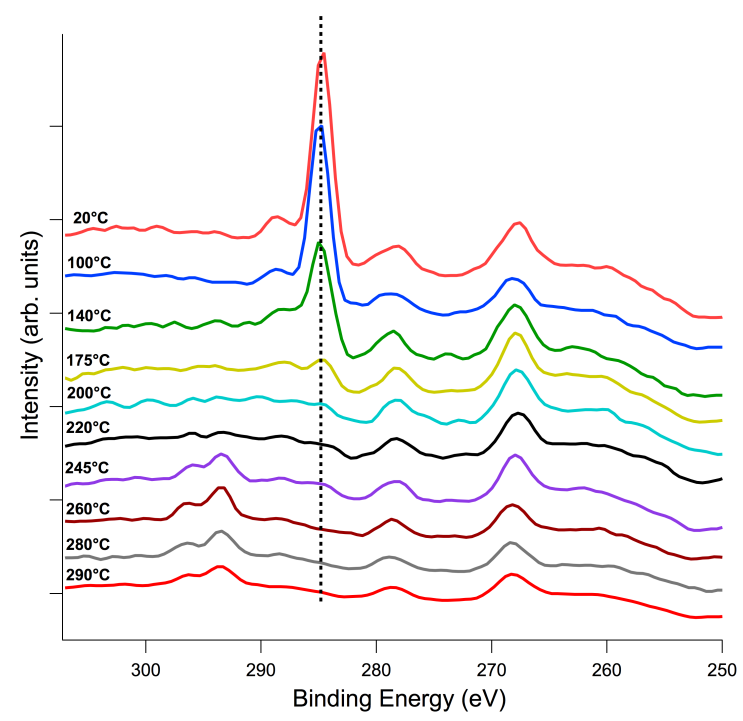

Figure 2: (Color available online) XPS spectra showing C $1 s$ and Sr $3 p$ core levels after successive anneals in $10^{-8}$ torr partial pressure of argon. Surface carbon appears $\sim 285 \mathrm{eV}$; a dotted line is provided as a guide to the eye. 


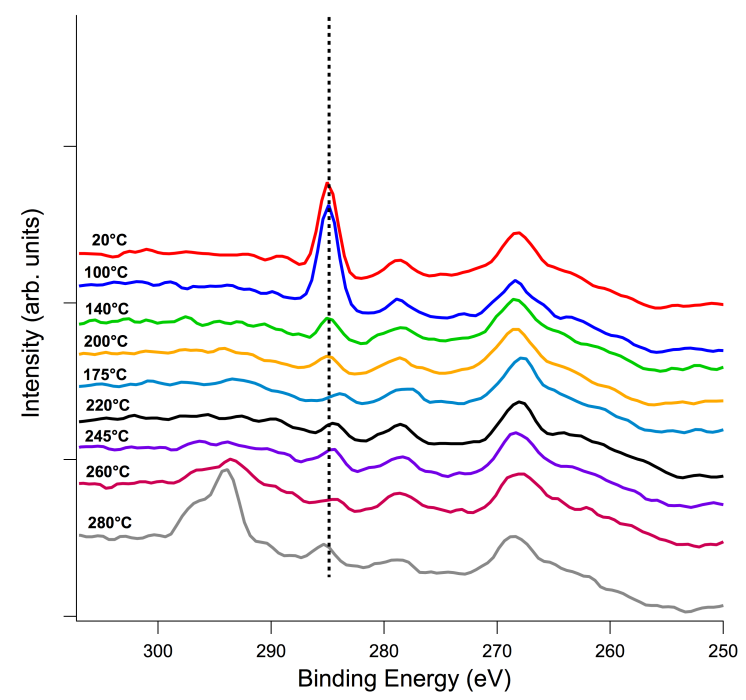

Figure 3: (Color available online) XPS spectra showing C $1 s$ and Sr $3 p$ core levels after successive anneals in $10^{-8}$ torr partial pressure of oxygen. Spectra are normalized to the Sr $3 p$ edge for comparison with Figure 2 

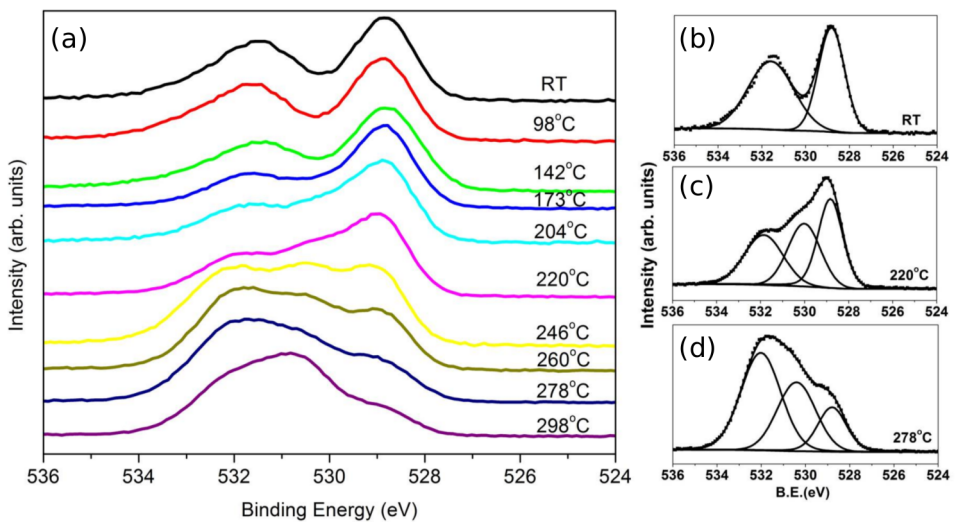

Figure 4: (Color available online) Oxygen $1 s$ spectra (a) for argon-annealed films. Typical multi-peak fits are shown in (b-d).Fits were performed using Voigt peaks with a Shirley background. 


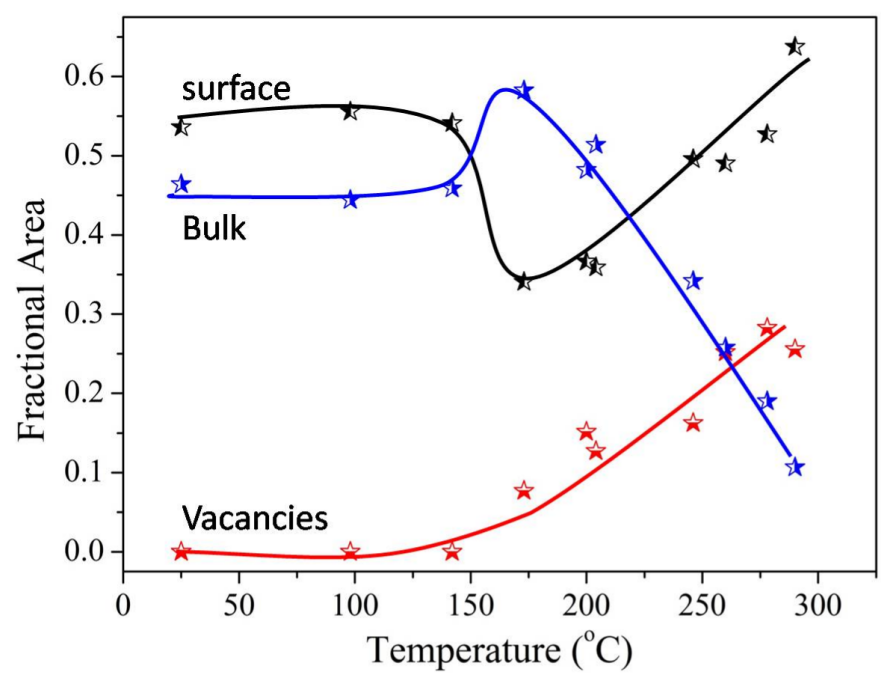

Figure 5: (Color available online) O $1 s$ core level multi-peak Voigt fit fractional areas, argon-annealed film. Solid lines are provided as guides to the eye. 

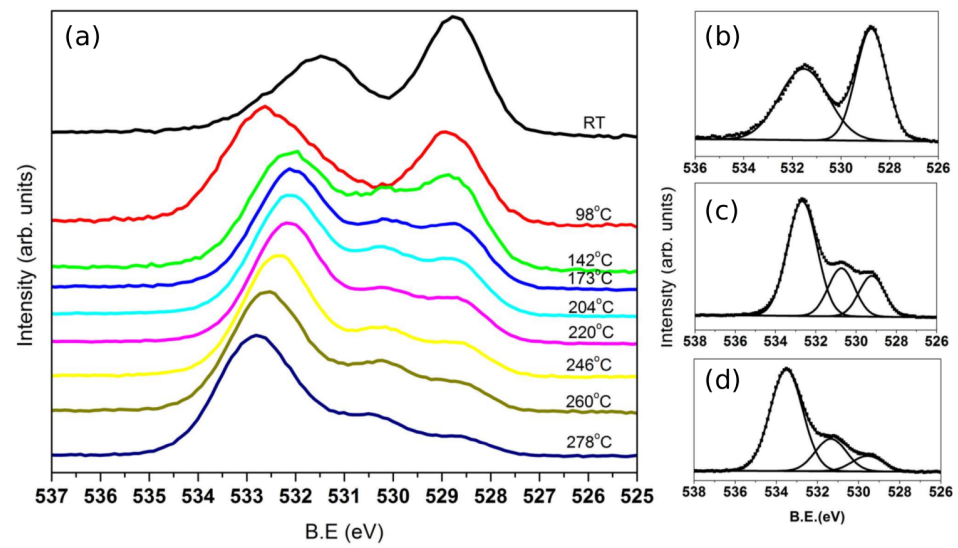

Figure 6: (Color available online) Oxygen $1 s$ spectra (a) for oxygen-annealed films. Typical multi-peak fits are shown in (b-d).Fits were performed using Voigt peaks with a Shirley background. 


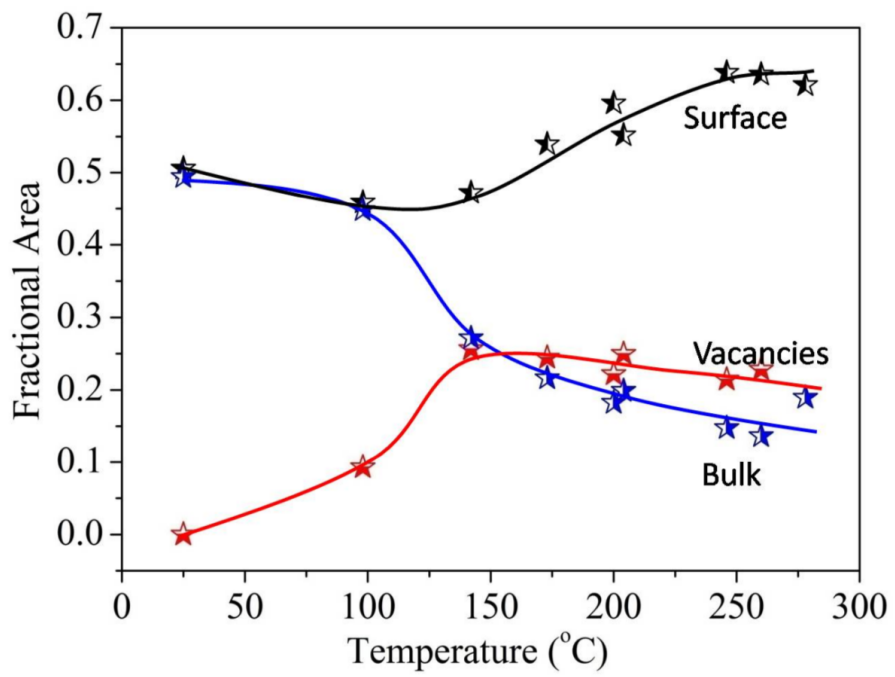

Figure 7: (Color available online) O1 $s$ core level multi-peak Voigt fit fractional areas, oxygen-annealed film. Solid lines are provided as guides to the eye. 


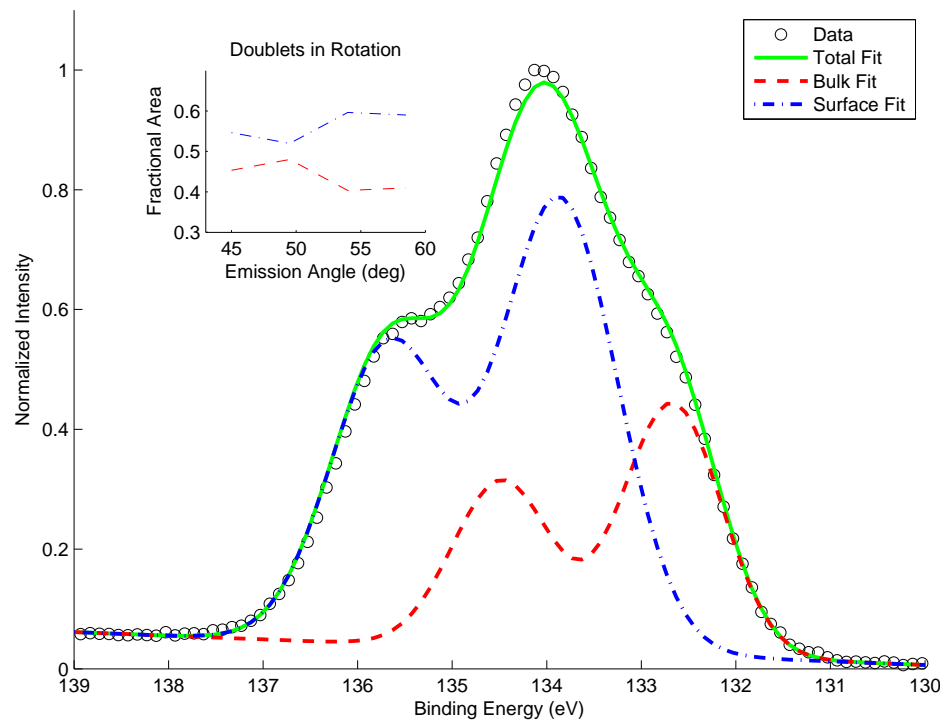

Figure 8: (Color available online) Typical strontium 3d core level for LSCF-6428. Best fit for two Gaussian doublets with linear background plotted against data. Inset shows the determination of surface vs. bulk states by angular surface sensitivity increase; as the surface angle with respect to the spectrometer increases, the measurement is more surface sensitive. 

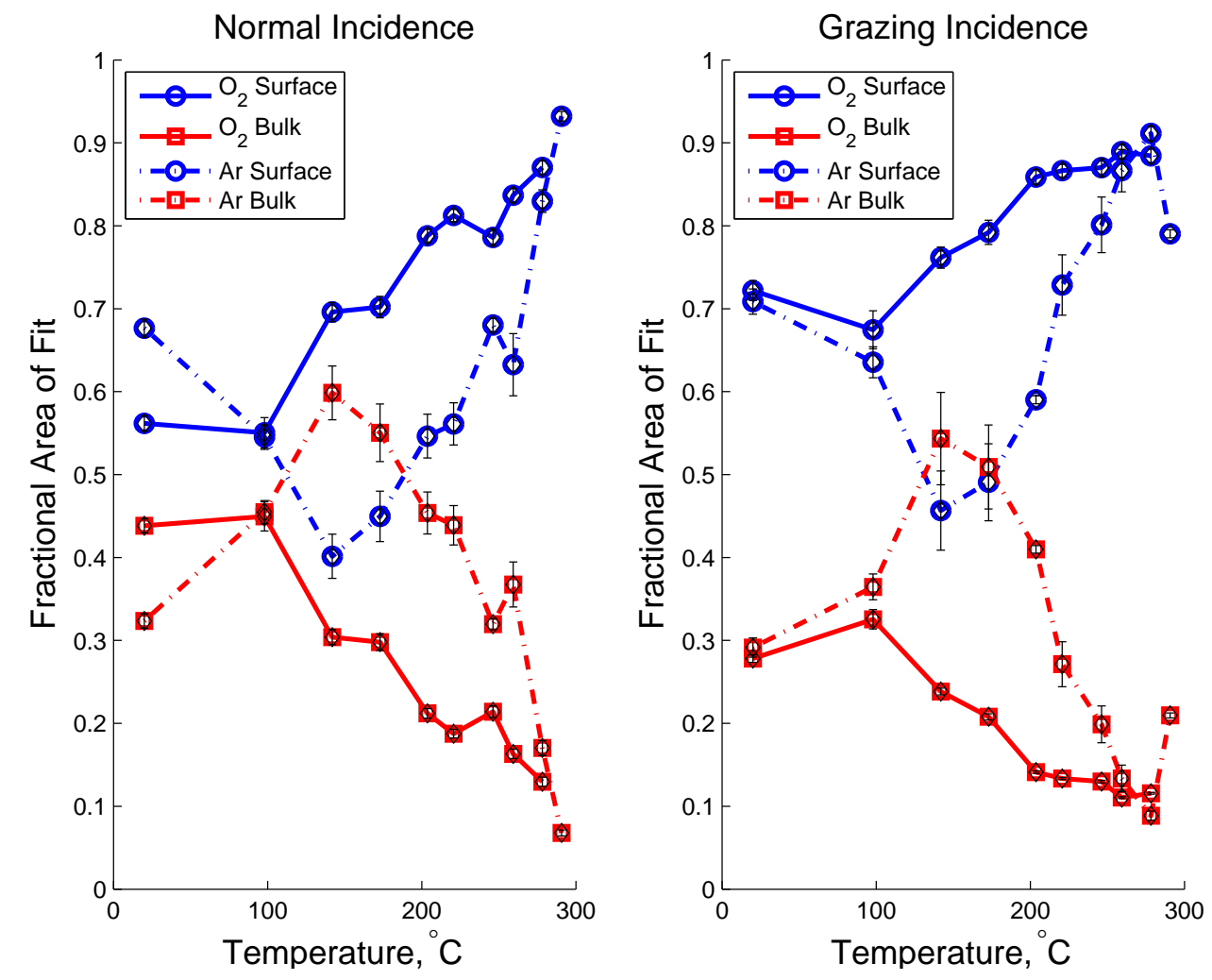

Figure 9: (Color available online) Fractional areas of the surface/bulk strontium phases over successive heating cycles, at (a) normal and (b) grazing incidence. Grazing incidence is more surface-sensitive. Error bars reflect the statistical uncertainty in the fits from which these fractions were calculated. 


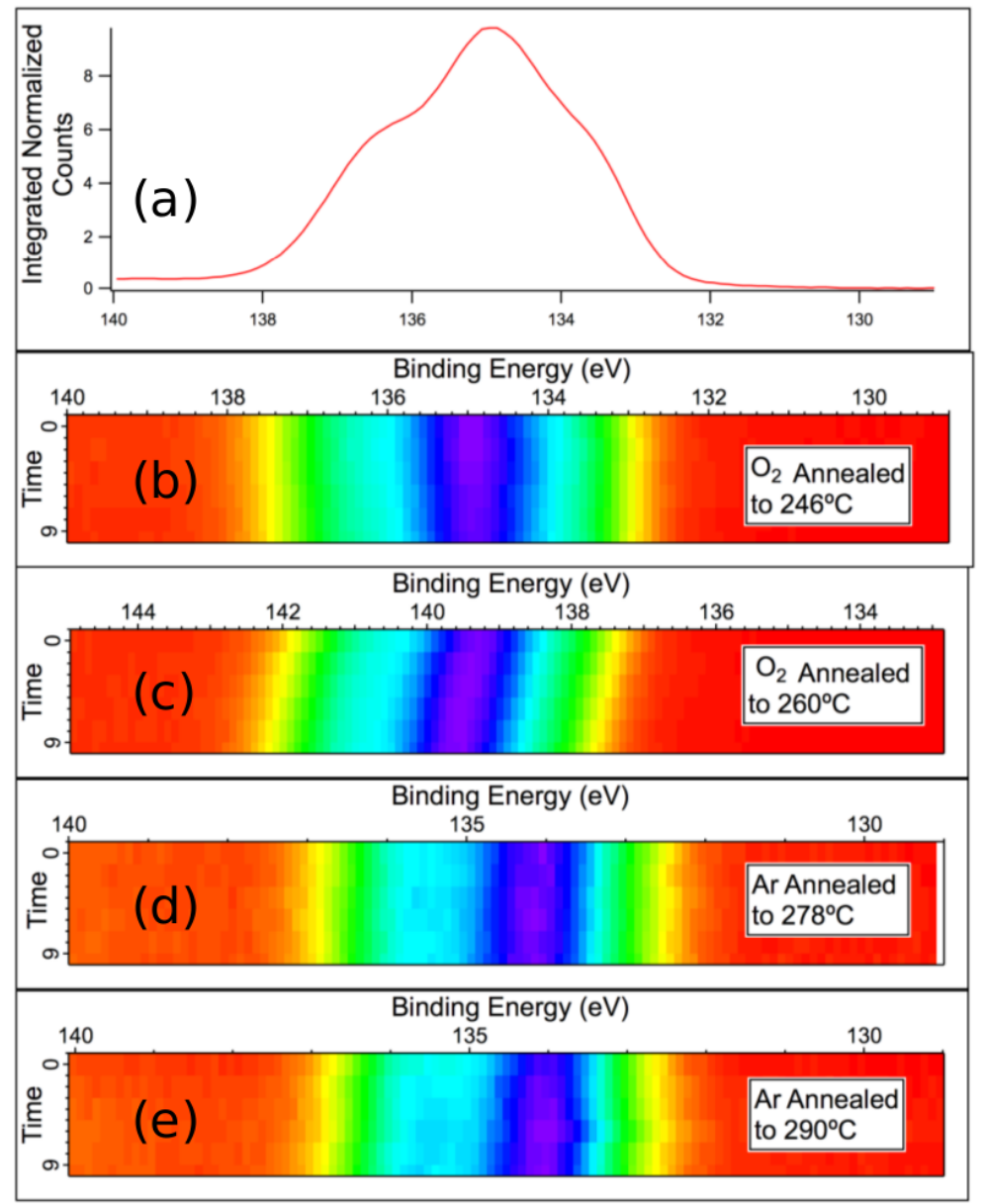

Figure 10: (Color available online) Time-dependent scans of Sr $3 d$ core levels. Time per scan is approximately 45 seconds. From top to bottom: (a) A plot showing integrated intensity over time for a well-conducting sample. (b) A time-dependent scan of a film annealed in $\mathrm{O}_{2}$ at $246{ }^{\circ} \mathrm{C}$, in which time proceeds down the y-axis. (c) A time-dependent scan clearly showing charge buildup over time after O 2 annealing at $260{ }^{\circ} \mathrm{C}$. (d), (e) Two more scans, of argon-annealed films annealed to over $278^{\circ} \mathrm{C}$, showing little charge buildup. 